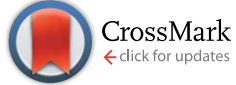

Cite this: RSC Adv., 2016, 6, 114505

\title{
Mixed matrix membranes prepared from non-dried MOFs for $\mathrm{CO}_{2} / \mathrm{CH}_{4}$ separations $\uparrow$
}

\begin{abstract}
Aylin Kertik, ${ }^{a}$ Asim Laeeq Khan ${ }^{\mathrm{b}}$ and Ivo F. J. Vankelecom*a
Mixed matrix membranes (MMMs) aim at combining the processibility of polymers with the molecular sieving of fillers to improve gas separation performance. Metal-organic frameworks (MOFs) are a new family of materials with promising potential as fillers. The first part of this work reports on exploiting the versatility of MOF synthesis routes by forming ZIF- 8 particles in polymer solutions to subsequently cast membranes directly from the solution. Although MOFs can be synthesised in a polymer medium, the decline in the synthesis yield does not allow for high loading in the MMMs. The second part describes a method for preparing MMMs with the commercial polyimide (PI) Matrimid ${ }^{\circledR}$ and ZIF-8, ZIF-7 and $\mathrm{NH}_{2}-\mathrm{MIL}-53(\mathrm{Al})$ as non-dried filler with $30 \mathrm{wt} \%$ and $50 \mathrm{wt} \%$ loading. A comparison of this method with the conventional approach of drying MOFs prior to incorporation exhibits the flexibility MOFs provide in membrane synthesis, in contrast to e.g. zeolites which intrinsically have to be calcined to become useful. The membranes with non-dried MOFs show some improvement in performance as compared to the unfilled polymer-only membranes, while those with dried MOFs even lose the inherent selectivity of the polymer.
\end{abstract}

Received 14th September 2016 Accepted 28th November 2016

DOI: 10.1039/c6ra23013j

www.rsc.org/advances the membrane, diffuses across the cross-section, and finally desorbs into the downstream. ,12,13 $^{\mathbf{1 2}}$ For fillers with a pore size relative to the kinetic diameter of penetrating gases, the diffusion of smaller gases is accelerated whereas larger gases cannot enter the pores. This size exclusion mechanism is known as molecular sieving. ${ }^{12-14}$ Over the last decades, porous fillers ranging from carbon molecular sieves ${ }^{15,16}$ and carbon nanotubes ${ }^{17-19}$ to ordered mesoporous silica ${ }^{20,21}$ and zeolites ${ }^{22-25}$ have been used in MMMs to provide molecular sieving to separate gases according to size and shape. ${ }^{26}$ Non-porous fillers are used to manipulate the polymer chain packing and modify the free volume..$^{27,28}$ Conventionally, a MMM is fabricated by dispersing the filler particles in a solvent, followed by adding polymer and mixing further to obtain a homogeneous solution. This solution is then cast to be dried or annealed. The dispersion of filler and the polymer-zeolite adhesion are notorious problems in membrane preparation. ${ }^{11}$ Poor dispersion is often created by post-synthesis calcination of fillers for the removal of templates, which leads to the irreversible formation of strong particle agglomerates. ${ }^{29,30}$ It is possible to break down these agglomerates to some extent by strong mixing methods, ${ }^{\mathbf{3 1}, 32}$ but these methods make the fabrication complicated and inefficient in terms of time and energy. The intrinsic lack of affinity between the inorganic and organic phases causes the formation of non-selective voids that lead to significant losses in selectivity. ${ }^{33}$ Many solutions suggested to promote adhesion, such as using compatibilisers, ${ }^{34,35}$ silylation, ${ }^{36}$ priming $^{.37,38}$ etc., have proven to be material- and time-intensive, and sometimes fail to enhance dispersion.

Recently, metal organic frameworks (MOFs) have stirred up excitement for MMMs, as well as for catalysis, ${ }^{39}$ sensing, $^{40}$ Lahore, Pakistan

$\dagger$ Electronic supplementary information (ESI) available. See DOI: 10.1039/c6ra23013j 
magnetism, ${ }^{41}$ semiconductors, ${ }^{42}$ and drug delivery. ${ }^{43}$ For gas separation, MOFs are very attractive owing to their tailorable chemistry, tunable composition, well-defined pore size, pore flexibility, and breathing effects. ${ }^{44}$ They consist of metal ions bridged with organic linkers that form a porous framework. ${ }^{44}$ Contrary to inorganic fillers, the organic linkers in MOFs offer better adhesion to the polymer. ${ }^{\mathbf{1 4}}$ Unfortunately, MOFs are not completely free of aggregation. ZIF-8 nanocrystals can stay in stable colloidal dispersion before drying, but are not redispersible after drying. ${ }^{45}$ Ultrasonication can distort the shape, size distribution, and structure of the MOF particles. ${ }^{46}$ Recent reports ${ }^{4-49}$ suggest storing MOFs in colloidal state to prevent the formation of strong covalent bonds between the particles. In another study, a one-pot approach for preparing MMMs by adding the polymer directly into the MOF synthesis liquor after MOF crystallisation, was reported. ${ }^{50}$

For this work, Matrimid®, a well-known commercial polyimide with properties that fall close to the Robeson upperbound ${ }^{51,52}$ was chosen as polymer. As filler, three different MOFs, namely ZIF-8, ZIF-7 and $\mathrm{NH}_{2}$-MIL-53(Al), were selected. ZIF-8 is composed of $\mathrm{Zn}$ (II) ion clusters linked by imidazolate ligands, with pores of $3.4 \AA$ in diameter. The pores are easily accessible to smaller gases such as $\mathrm{CO}_{2}$ and $\mathrm{H}_{2}{ }^{51} \mathrm{ZIF}-7$ belongs to the same family as ZIF-8, and exhibits a sodalite topology formed by connecting zinc clusters with benzimidazole. ${ }^{48} \mathrm{ZIF}-7$ has an estimated pore size of $3 \AA{ }^{53}$ MIL-53(Al) consists of $\mathrm{AlO}_{4}(\mathrm{OH})_{2}$ octahedra and 1,4-benzenedicarboxylate (terephthalate) linkers, ${ }^{54}$ which can be amino-functionalized to form $\mathrm{NH}_{2}$-MIL-53(Al) with amine moieties on the surface. ${ }^{55}$ This MOF exhibits a so-called breathing behaviour by changing from a narrow pore (np) structure to a large pore (lp) structure at high partial pressures of $\mathrm{CO}_{2}{ }^{56,57}$

Two novel preparation methods are designed in this work to prepare high-loading MMMs, as well as to simplify and optimise earlier approaches. The first part focuses on the synthesis of MOF particles inside a polymeric solution. This solution with perfectly dispersed MOFs will then be used directly as membrane casting solution. A second part investigates the difference between MMMs prepared with the conventional method and the so-called "wet-MOF method".

\section{Experimental}

\section{Materials}

Polyimide (Matrimid $® 5218$ ) was kindly provided by Huntsman (Switzerland) and used after drying at $110{ }^{\circ} \mathrm{C}$ overnight.

For ZIF-8 synthesis, zinc nitrate hexahydrate $\left(\mathrm{Zn}\left(\mathrm{NO}_{3}\right)_{2}\right.$ $\left.\cdot 6 \mathrm{H}_{2} \mathrm{O}\right)$ and 2-methylimidazole $\left(\mathrm{C}_{4} \mathrm{H}_{6} \mathrm{~N}_{2}\right)$ were obtained from Acros Organics (Belgium). Triethylamine $\left(\mathrm{C}_{6} \mathrm{H}_{15} \mathrm{~N}\right)$ was purchased from Merck (Germany).

For ZIF-7 synthesis, benzimidazole $\left(\mathrm{C}_{7} \mathrm{H}_{6} \mathrm{~N}_{2}\right)$ was obtained from Acros Organics (Belgium).

For $\mathrm{NH}_{2}$-MIL-53(Al) synthesis, 2-aminoterephthalic acid $\left(\left(\mathrm{H}_{2} \mathrm{NC}_{6} \mathrm{H}_{3}-1,4-\left(\mathrm{CO}_{2} \mathrm{H}\right)_{2}\right)\right.$ and aluminum nitrate nonahydrate $\left(\mathrm{Al}\left(\mathrm{NO}_{3} \cdot 9 \mathrm{H}_{2} \mathrm{O}\right)\right.$ were obtained from ChemLab and Acros Organics, respectively.

Dimethylformamide $\left(\left(\mathrm{CH}_{3}\right)_{2} \mathrm{NCOH}, \quad \mathrm{DMF}\right)$, chloroform $\left(\mathrm{CHCl}_{3}\right)$, and methanol $\left(\mathrm{CH}_{3} \mathrm{OH}\right)$ were purchased from Acros
Organics (Belgium) and VWR (Belgium), and used for solvent exchange and membrane preparation. All solvents were dried overnight using activated zeolite $4 \mathrm{~A}$ beads prior to use.

\section{In situ synthesis of MOF particles}

ZIF-8@Matrimid® membranes were prepared in situ by adding MOF precursors inside a dilute solution ( $5 \mathrm{wt} \%$ ) of Matrimid $®$ in DMF at $20,40,60$ or $80{ }^{\circ} \mathrm{C}$. In contrast to a previous report on a one-pot synthesis method, ${ }^{\mathbf{5}}$ this approach refers to crystallising MOF particles in a polymeric solution. The ZIF-8 synthesis recipe was adapted from ref. 51 with a 2-methylimidazole (2-mim) to $\mathrm{Zn}\left(\mathrm{NO}_{3}\right)_{2}$ ratio of $8: 1$. This modification was applied in order to overcome the brittleness caused by the unreacted $\mathrm{Zn}\left(\mathrm{NO}_{3}\right)_{2}$ that remains in the membrane. Fig. $1 \mathrm{a}$ and $\mathrm{b}$ show membranes prepared by adding $20 \mathrm{wt} \%$ of $\mathrm{Zn}\left(\mathrm{NO}_{3}\right)_{2}$ and 2-mim to Matrimid ${ }^{\circledR}$, respectively. While Matrimid® with 2-mim formed a homogeneous, standalone membrane, the membrane with $\mathrm{Zn}\left(\mathrm{NO}_{3}\right)_{2}$ was brittle and broken. The recipe was downscaled to match the amount of DMF in the polymer solution $(10 \mathrm{~mL})$. Unlike the original procedure, the synthesis compounds were added directly into the Matrimid ${ }^{\circledR}$ solution. The mixture was stirred for 4 days to give enough time for ZIF-8 synthesis, which was then poured into a Petri dish to dry at $100{ }^{\circ} \mathrm{C}$ until the membrane solidified.

In order to increase the amount of ZIF-8 particles in the Matrimid® solution, the concentration of the synthesis components was increased to give 13, 33 and $53 \mathrm{wt} \%$ loading, as calculated based on the yield reported in ref. 51 .

\section{Preparation of MMMs with wet MOFs}

ZIF-8 was synthesised as described in ref. 51. Upon cooling, the particles were washed with DMF, methanol and $\mathrm{CHCl}_{3}$, respectively.

ZIF-7 was synthesised by dissolving zinc nitrate hexahydrate $(0.302 \mathrm{~g})$ and benzimidazole (0.769 g) in DMF (400 mL), and magnetically stirring the mixture for $48 \mathrm{~h}$ at room temperature. The particles were washed with DMF, methanol, $\mathrm{CHCl}_{3}$, respectively.

$\mathrm{NH}_{2}$-MIL-53(Al) was synthesised by dissolving 2-aminoterephthalic acid $(2 \mathrm{~g})$ and $\mathrm{Al}\left(\mathrm{NO}_{3}\right)_{3} \cdot 9 \mathrm{H}_{2} \mathrm{O}(2 \mathrm{~g})$ in distilled water $(400 \mathrm{~mL})$. The solution was heated to $100{ }^{\circ} \mathrm{C}$ for $6 \mathrm{~h}$. Upon cooling, the particles were centrifuged and washed with DMF, methanol and $\mathrm{CHCl}_{3}$, respectively.
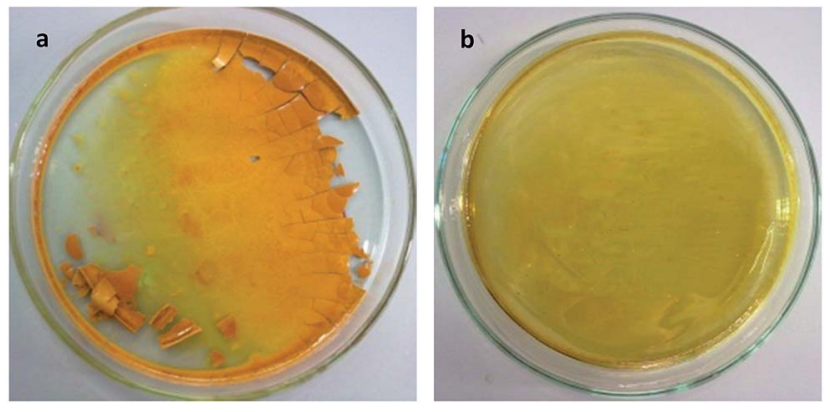

Fig. 1 Matrimid $®$ with $20 \mathrm{wt} \%$ (a) $\mathrm{Zn}\left(\mathrm{NO}_{3}\right)_{2}$ and (b) 2-mim. 
At the end of the synthesis procedure, the MOF particles were washed as detailed below, and the remaining sludge was separated into two equal parts, one part was dried in the oven overnight $\left(240{ }^{\circ} \mathrm{C}\right.$ for ZIF-8, $100{ }^{\circ} \mathrm{C}$ for ZIF-7 and $\mathrm{NH}_{2}$-MIL$53(\mathrm{Al})$ ), and the other part was kept in $\mathrm{CHCl}_{3}$ to be directly used for membrane fabrication.

In order to prepare a membrane solution, the MOF dispersion and Matrimid ${ }^{\circledR}$ were mixed in certain amounts to achieve the desired membrane loading, as shown in eqn (1), and stirred magnetically overnight. When a homogenous mixture was obtained, it was poured into a Petri dish and dried at room temperature under $\mathrm{N}_{2}$ until the membrane solidified. The solidified membranes were gradually heated to $100{ }^{\circ} \mathrm{C}$ and dried for two days, allowing to cool down gradually prior to their removal from the oven.

$$
\text { Loading }\left(w t^{\%} \%\right)=w t_{\text {MOF }} /\left(w t_{M O F}+w t_{\text {polymer }}\right)
$$

\section{Preparation of conventional MMMs}

The membranes with MOF loadings of 1, 3, 6, 10 and 13 wt\% were prepared by dispersing the particles in DMF followed by the addition of Matrimid ${ }^{\circledR}$ and casting the solution into a Petri dish. Membranes with 30 and 50 wt\% dried MOF loading were prepared by dispersing the particles in $\mathrm{CHCl}_{3}$. All membranes were dried at $100{ }^{\circ} \mathrm{C}$ for two days, allowing to cool down gradually prior to their removal from the oven.

\section{Characterisation}

The morphology of membrane cross-sections was observed using a JEOL JSM-1060LV. The SEM samples were prepared by freeze-fracturing in liquid $\mathrm{N}_{2}$. In order to prevent charge buildup, the samples were sputtered with $\mathrm{Au} / \mathrm{Pd}$ for three cycles of $20 \mathrm{~s}$.

X-ray diffraction patterns were obtained using a Stoe-HT X-ray diffractometer with $\mathrm{CuK} \alpha$ radiation $(\lambda=1.54 \AA)$ at room temperature.

The gas separation performance of a membrane is determined by the permeability of penetrants, and the separation factor. The rate at which gas molecules permeate through a membrane is the permeability coefficient, defined as the product of the diffusion and solubility coefficients, as shown in eqn (2), where $P_{i}, D_{i}$ and $S_{i}$ are the permeability, diffusion and solubility coefficients of species $i$, respectively.

$$
P_{i}=D_{i} S_{i}
$$

The selectivity of a membrane for pure gases (ideal selectivity) is the ratio of the permeation coefficient of component $i$ to that of component $j$, as given in eqn (3), where $\alpha_{i / j}$ is the ideal selectivity for $i$ over $j$, and $P_{i}$ and $P_{j}$ are the permeabilities of $i$ and $j$, respectively.

$$
\alpha_{i / j}=P_{i} / P_{j}
$$

Pure-gas selectivity fails to provide a real-life estimation of membrane performance, especially when highly soluble gases (such as $\mathrm{CO}_{2}$ ) are concerned. The mixed gas selectivity is more realistic, as it is obtained with a mixed feed and competitive sorption is also taking place during permeation. Mixed gas selectivity is calculated as given in eqn (4), where $y_{i}, y_{j}$ are the mole fractions of $i$ and $j$ in the permeate, and $x_{i}$ and $x_{j}$ are the mole fractions of $i$ and $j$ in the feed, respectively.

$$
\alpha_{i l j}=\left(y_{i} / y_{j}\right) /\left(x_{i} / x_{j}\right)
$$

The gas separation performance for binary gas mixtures was tested using a custom-built, high-throughput gas separation system (HTGS), described in detail in ref. 58. A mixed gas feed of $50-50 \%$ vol. $\mathrm{CO}_{2}-\mathrm{CH}_{4}$ at $35{ }^{\circ} \mathrm{C}$ and 10 bar cross-membrane pressure difference was used. The composition of the permeate side was analysed by gas chromatography (GC) until three consecutive measurements gave the same result, which indicates steady-state. The reported selectivities are the average of these three measurements. Permeabilities were measured using the constant-volume variable-pressure method after steady-state was ensured.

\section{Results and discussion}

\section{Synthesis of ZIF-8 particles in a PI-solution}

ZIF-8 particles were synthesised in a dilute solution of Matrimid® by directly adding $\mathrm{Zn}\left(\mathrm{NO}_{3}\right)_{2} \cdot 6 \mathrm{H}_{2} \mathrm{O}$ and 2-methylimidazole to it, and stirring the mixture at $20,40,60$ or $80{ }^{\circ} \mathrm{C}$ for 4 days. As seen in Fig. 2, no ZIF-8 particles could be observed in the cross-sections of the membranes prepared at 20 or $40{ }^{\circ} \mathrm{C}$, but some particles could be observed for samples prepared at $60{ }^{\circ} \mathrm{C}$ and $80^{\circ} \mathrm{C}$. As a result of the in situ synthesis approach, these particles are perfectly distributed in the polymer matrix.

The XRD spectra of the MMMs (Fig. 3a) did not exhibit the characteristic peaks of ZIF-8. Due to the presence of unreacted $\mathrm{Zn}$-source in the membranes, TGA could not be used to determine the ZIF-8 loading based on the Zn content. ${ }^{59}$ Instead, the XRD spectra of conventional MMMs with pre-synthesised MOFs at loadings of 1, 3, 6, 10 and $13 \mathrm{wt} \%$ were used for comparison, (Fig. 3b). These membranes were prepared by dispersing presynthesised and dried MOF particles in a solvent and adding the polymer to this mixture. As the characteristic peaks of ZIF-8 started to appear only from $6 \mathrm{wt} \%$ loading onward for these samples, the ZIF- 8 loading in the in situ prepared MMMs was thus assumed to be below $6 \mathrm{wt} \%$, and hence much below the limits of application. In an attempt to increase the ZIF-8 loading, the concentration of MOF precursors in the polymer solution was increased to achieve up to a theoretical $53 \mathrm{wt} \%$ of MOF loading, based on a $48 \%$ conversion of the precursors. In addition to gelation of this mixture and the failure to form standalone layers, these membranes also did not show any indication of significant loading (Fig. 4 and 5); with the exception of a $\mathrm{Zn}(\mathrm{OH})_{2}$ peak around $27-28^{\circ}$ that signifies low crystallinity ${ }^{60}$ (Fig. 4b). Therefore, it was clear that changing the synthesis medium from a solvent to a polymer solution was detrimental to the synthesis yield, regardless of the concentration of the precursors. 

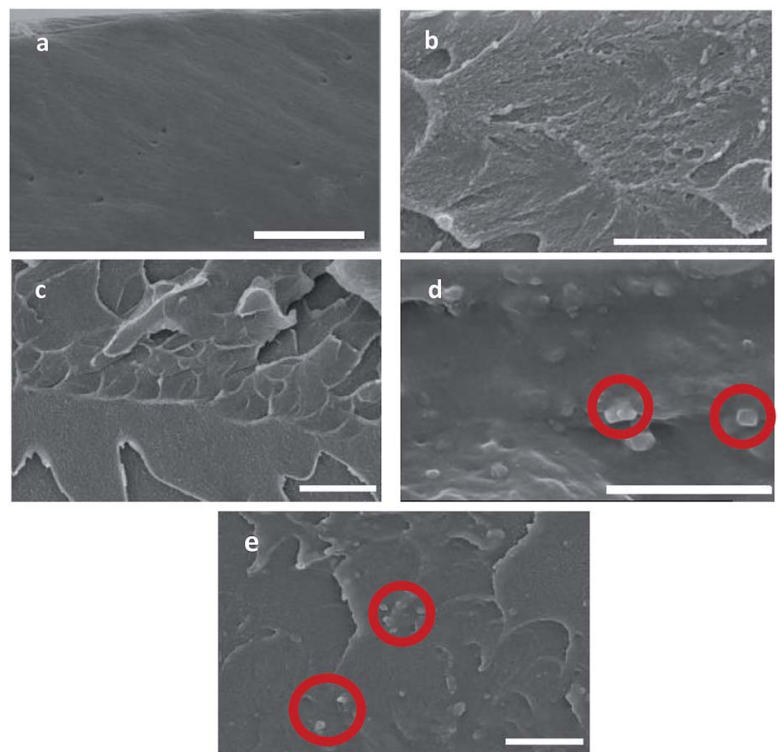

Fig. 2 SEM images of (a) Matrimid $®$, and ZIF-8@Matrimid ${ }^{\circledR}$ MMMs prepared at (b) $20^{\circ} \mathrm{C}$, (c) $40{ }^{\circ} \mathrm{C}$, (d) $60^{\circ} \mathrm{C}$ and (e) $80^{\circ} \mathrm{C}$. The bars represent $5 \mu \mathrm{m}$. The red circles show ZIF- 8 particles.

\section{Non-dried vs. dried MOFs}

The membrane preparation method was changed to omitting post-synthesis drying, similar to ref. $47,49,61$ and 62 . In order to demonstrate the effectiveness of this method, membranes with dried MOFs were also prepared for comparison. Comparability was assured by preparing the MMMs with $\mathrm{MOF}^{\mathrm{dr}}$ by using the exact method as for $\mathrm{MOF}^{\mathrm{n}-\mathrm{dr}}$, i.e. only magnetic
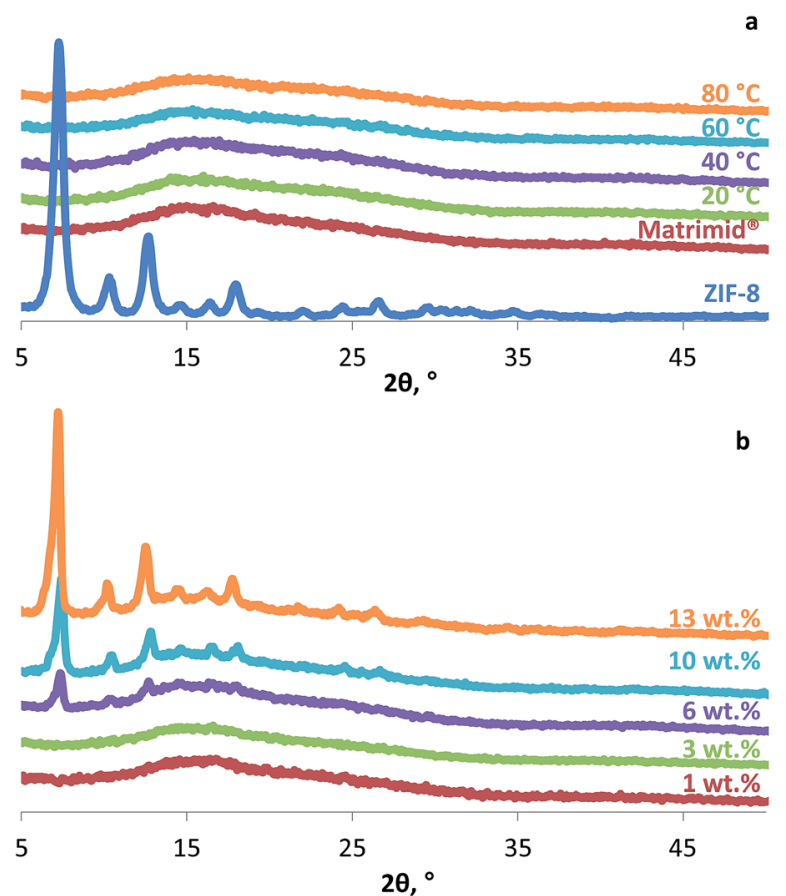

Fig. 3 XRD spectra of (a) ZIF-8@Matrimid® prepared at 20,40,60 and $80^{\circ} \mathrm{C}$, and (b) membranes with controlled loading of dried ZIF- 8 .
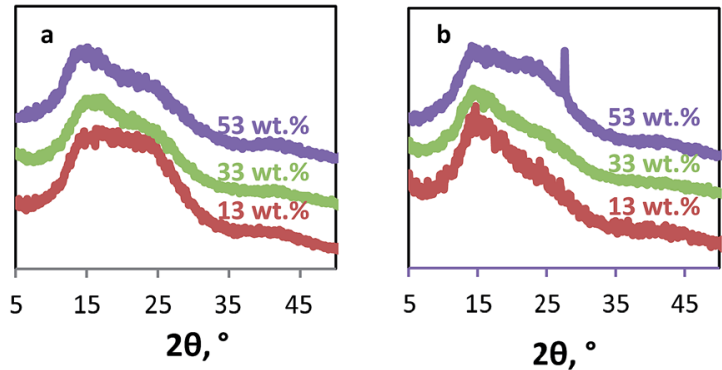

Fig. 4 XRD spectra of MMMs with increased precursor concentration to achieve theoretical loadings of 13,33 and $53 \mathrm{wt} \%$, synthesised at (a) $20^{\circ} \mathrm{C}$ and (b) $60{ }^{\circ} \mathrm{C}$.

stirring was applied to distribute the $\mathrm{MOF}^{\mathrm{dr}}$ particles. The crosssectional SEM images (Fig. 6) exhibit the clear difference between MMMs with dried and non-dried MOFs (from now on $\mathrm{MOF}^{\mathrm{n}-\mathrm{dr}}$ for non-dried and $\mathrm{MOF}^{\mathrm{dr}}$ for dried MOFs, respectively). $\mathrm{MOFs}^{\mathrm{dr}}$ formed big agglomerates that were completely detached from the polymer, especially at high loadings. On the contrary, the MOFs ${ }^{\mathrm{n}-\mathrm{dr}}$ were perfectly distributed and embedded in the polymer, despite the very high loadings (30 and $50 \mathrm{wt} \%$ ).

As shown in ESI Fig. $1, \uparrow$ the XRD spectra of the MMMs were in agreement with their respective MOFs for both the wet and dry synthesis method. The spectra show that the MOF particles remained intact during membrane preparation (thanks to the mild mixing conditions); as opposed to the work in ref. 46, where high-intensity ultrasonication of MOF particles resulted in changes in the structure of ZIF-8 particles.

\section{Gas separation performance}

Gas separation performance is a direct indicator of the presence or absence of microdefects, ${ }^{63}$ and the good dispersion of MOFs in MMMs. There was an obvious difference between the gas separation performances of the MMMs with non-dried and dried MOFs, as given in Fig. 7. A more detailed table with reproducibility data can be found in ESI Table $1 . \dagger$

The MMM with $30 \mathrm{wt} \%$ ZIF-8 ${ }^{\mathrm{n}-\mathrm{dr}}$ exhibited a tripled $\mathrm{CO}_{2}$ permeability compared to the unfilled polymer along with a slight decrease in selectivity from 22.3 to 18.6. The increased permeation of $\mathrm{CO}_{2}$ compared to $\mathrm{CH}_{4}$ can be attributed to the intrinsic framework flexibility of ZIF-8 ${ }^{64-66}$ which allows for the faster diffusion of both gases. Although it belongs to the same family as ZIF-8, ZIF-7 ${ }^{\text {n-dr }}$ exhibited a selectivity increase over unfilled Matrimid $\circledast$ and a small increase in permeability. Also,

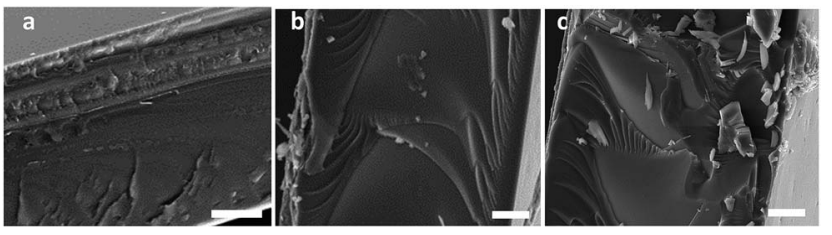

Fig. 5 Cross-sectional SEM images of MMMs with increased precursor concentration to achieve a theoretical loading of (a) 13 (b) 33 (c) 53 $w t \%$. The bars represent $10 \mu \mathrm{m}$. 

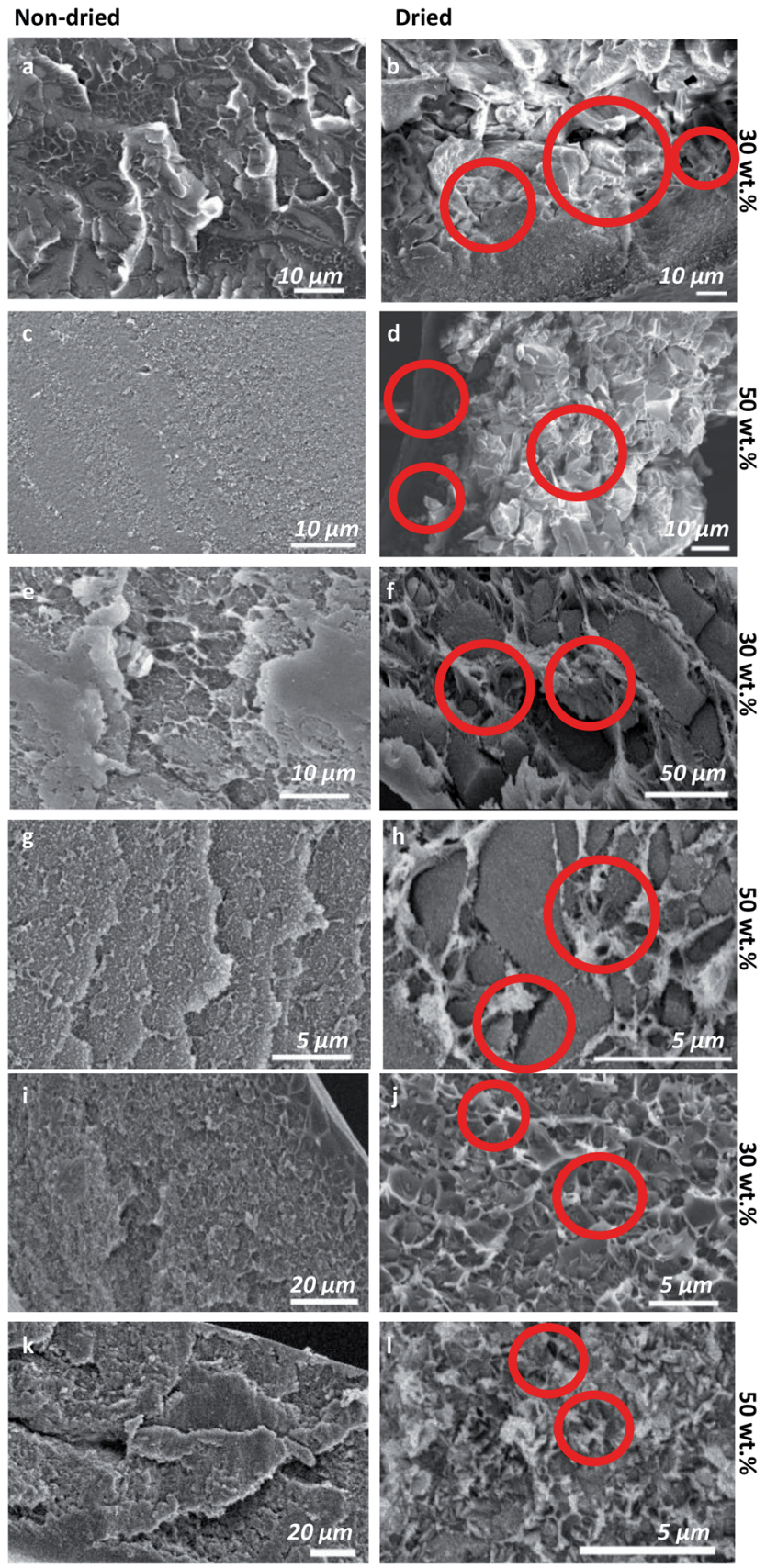

Fig. 6 SEM images of MMMs with (a-d) ZIF-8, (e-h) ZIF-7 and (i-l) $\mathrm{NH}_{2}-$ MIL-53(Al). The red circles mark the defective interface between the Matrimid ${ }^{\circledR}$ matrix and the agglomerated MOF particles.

both MOFs undergo similar guest-induced "gate opening", ${ }^{7,68}$ but their intrinsic pore apertures are different: ZIF-8 has a larger pore aperture of $3.4 \AA$ versus $3.0 \AA$ for ZIF-7. In addition, it has been reported that ZIF-8 is much more susceptible to the enlargement of the pore aperture via the "gate opening" phenomenon. ${ }^{69}$ Therefore, the ZIF- ${ }^{\mathrm{n}-\mathrm{dr}}$ and ZIF- ${ }^{\mathrm{n}-\mathrm{dr}}$ showed opposite improvement in membrane performance as MMM fillers. Just like $\mathrm{ZIF}-7^{\mathrm{n}-\mathrm{dr}}$, the MMMs with $\mathrm{NH}_{2}$-MIL-53(Al) $)^{\mathrm{n}-\mathrm{dr}}$ also showed no change in permeability and a small increase in selectivity. In contrast to its unfunctionalised parent, the np form of $\mathrm{NH}_{2}-\mathrm{MIL}-53(\mathrm{Al})$ is preferred at low $\mathrm{CO}_{2}$ pressures (below

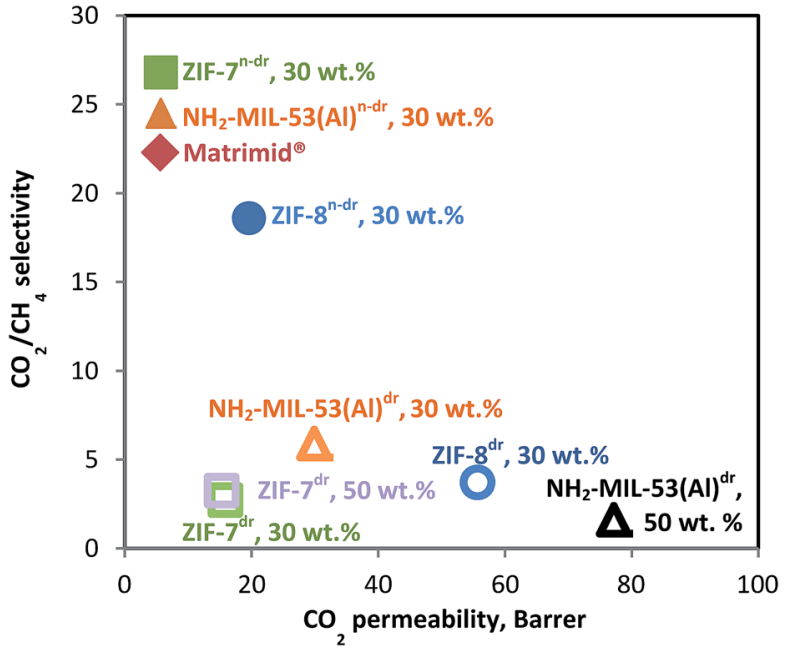

Fig. 7 Gas separation performance of MMMs with non-dried (filled symbols) and dried ZIF-8, ZIF-7 and $\mathrm{NH}_{2}$-MIL-53(Al) (open symbols).

10 bar (ref. 57)). Therefore, it is sensible that there is no significant increase in permeability for the measurement conditions of this work ( 5 bar). All in all, these MMMs show improved selective behaviour without sacrificing the intrinsic permeability of the polymer.

In agreement with the SEM results, the defects formed by the dried MOFs caused complete deterioration of the inherent selectivity of the polymer. The agglomerates that were formed as a result of drying, created non-selective voids that made the selectivity drop substantially. The permeabilities were increased up to 13 times. For a better comparison of the extent of change in selectivity, the enhancement ratio was calculated as the ratio of the selectivity of the MMM to that of the polymer (Fig. 8). The improvement in membrane performance followed the order ZIF- ${ }^{\mathrm{n}-\mathrm{dr}}>\mathrm{NH}_{2}-\mathrm{MIL}-53(\mathrm{Al})^{\mathrm{n}-\mathrm{dr}}>\mathrm{ZIF}-8^{\mathrm{n}-\mathrm{dr}}$ for $\mathrm{MOFs}^{\mathrm{n}-\mathrm{dr}}$ whereas the selectivity decay did not follow a certain order, but it clearly could not exceed 0.3 for MOFs ${ }^{\mathrm{dr}}$.

\section{Comparison with Maxwell model}

There are various theoretical expressions for predicting the gas separation performance of MMMs. An adaptation of the Maxwell model (eqn (5)), which was originally developed for calculating the electrical conductivity of composites, is

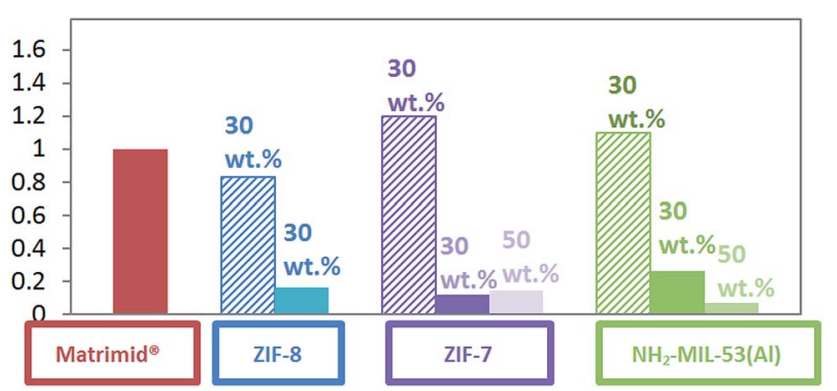

Fig. 8 Enhancement in selectivity with non-dried (striped bars) and dried (filled bars) MOFs. 

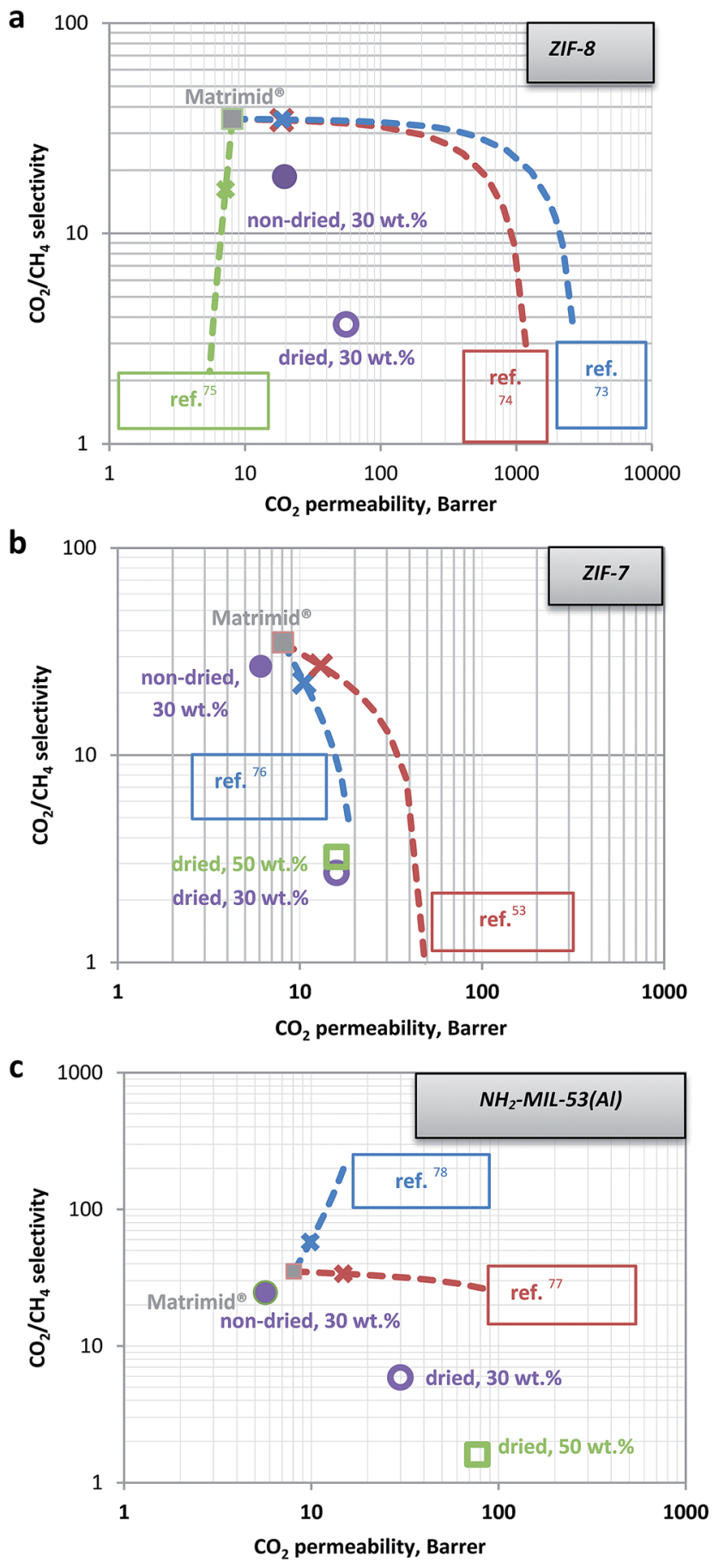

Fig. 9 Comparison of membrane performance with Maxwell predictions for (a) ZIF-8, (b) ZIF-7 and (c) $\mathrm{NH}_{2}-\mathrm{MIL}-53(\mathrm{Al})$. The filled and empty symbols represent the data from this work, with non-dried and dried MOFs, respectively. The dashed lines show the Maxwell predictions from 0 to 100 wt\% loading with the reference used for the 100 wt\% MOF performance mentioned in frames, from ref. 53, 73-78. The predictions for $30 \mathrm{wt} \%$ loading are marked with X's on the dashed lines.

conventionally used to estimate the permeability and selectivity of a MMM comprised of counterparts of known permeabilities. The Maxwell model assumes an ideal case without membrane defects, and without change in the separation properties of the counterparts.

$$
P_{\mathrm{r}}=\frac{P}{P_{\mathrm{m}}}=\left[\frac{2(1-\varphi)+(1+2 \varphi) \lambda_{\mathrm{dm}}}{(2+\varphi)+(1-\varphi) \lambda_{\mathrm{dm}}}\right]
$$

where $P_{\mathrm{r}}$ is the relative permeability of the permeant, $P$ is the effective permeability of the permeant in the MMM, $P_{\mathrm{m}}$ is the effective permeability of the permeant in the matrix (continuous phase), $\varphi$ is the volume fraction of filler particles, $\lambda_{\mathrm{dm}}$ is the ratio of permeability of the dispersed filler particles $\left(P_{\mathrm{d}}\right)$ to the permeability of the matrix $P_{\mathrm{m}}{ }^{70}$ The Maxwell model generally predicts the permeability well when $\varphi$ is less than about 0.2. Moreover, the Maxwell model cannot predict the correct behavior when $\varphi$ is close to the maximum packing volume fraction of filler particles. The model does not account for particle size distribution, particle shape, and aggregation of particles. $^{\text {7o }}$

Fig. 9 shows the comparison of the experimental results from this work and the Maxwell predictions based on different literature reports for MOF performances. Detailed information on the Maxwell predictions is given in ESI Tables S1-S3. $\dagger \mathrm{ZIF}-8^{\mathrm{n}-\mathrm{dr}}$ matches the permeability but cannot reach the selectivity predicted, whereas ZIF- $7^{\text {n-dr }}$ matches the predicted selectivity much better, but falls short on permeability. $\mathrm{NH}_{2}-\mathrm{MIL}-53(\mathrm{Al})^{\mathrm{n}-\mathrm{dr}}$ cannot reach the permeability and selectivity predicted, which may be related to the interaction between the amino groups of the MOF with the amide groups of the polymer chains. ${ }^{71,72}$

\section{Conclusions}

Two different approaches were applied to prepare MOFcontaining MMMs. First, ZIF-8 particles were synthesized by adding the MOF precursors directly into a dilute solution of Matrimid®. Although individual particles could be observed in the SEM images, XRD analysis revealed that the loading was too low for practical use. Increasing the concentration of the precursors did not achieve higher filler content. Moreover, the membranes were gelled by the unreacted precursors. Secondly, in order to increase and control MOF loading, MOFs were synthesized separately and embedded into the membrane in their non-dried form to prevent the formation of agglomerates during drying. The different properties of the MOFs resulted in different outcomes in terms of gas separation performance. ZIF- $8^{\text {n-dr }}$ showed an impressive increase in permeability along with a slight loss of selectivity, whereas $\mathrm{ZIF}-7^{\mathrm{n}-\mathrm{dr}}$ and $\mathrm{NH}_{2}$-MIL$53(\mathrm{Al})^{\mathrm{n}-\mathrm{dr}}$ exhibited increased selectivities and very small increases in permeability. Dried MOFs prepared using the same method were severely agglomerated and detached from the polymer to the extent that the inherent selectivity of Matrimid® was lost. Maxwell predictions overestimated the performances, probably due to the flexible nature of the MOFs.

\section{Acknowledgements}

A. K. is thankful to the Erasmus-Mundus Doctorate in Membrane Engineering Programme. We also would like to thank IAP funding of the Belgian Federal government and Huntsman (Switzerland) for kindly providing the Matrimid ${ }$. 


\section{References}

1 P. Pandey, Prog. Polym. Sci., 2001, 26, 853-893.

2 S. Sridhar, B. Smitha and T. M. Aminabhavi, Sep. Purif. Rev., 2007, 36, 113-174.

3 M. Shah, M. C. Mccarthy, S. Sachdeva, A. K. Lee and H. Jeong, Ind. Eng. Chem. Res., 2012, 51, 2179-2199.

4 L. M. Robeson, J. Membr. Sci., 1991, 62, 165-185.

5 L. M. Robeson, J. Membr. Sci., 2008, 320, 390-400.

6 S. Lagorsse, J. Membr. Sci., 2004, 241, 275-287.

7 F. Gallucci, E. Fernandez, P. Corengia and M. van Sint Annaland, Chem. Eng. Sci., 2013, 92, 40-66.

8 N. Kosinov, J. Gascon, F. Kapteijn and E. J. M. Hensen, J. Membr. Sci., 2016, 499, 65-79.

9 T. Chung, L. Jiang, Y. Li and S. Kulprathipanja, Prog. Polym. Sci., 2007, 32, 483-507.

10 T. Moore and W. Koros, J. Mol. Struct., 2005, 739, 87-98.

11 R. Mahajan, R. Burns, M. Schaeffer and W. J. Koros, J. Appl. Polym. Sci., 2002, 86, 881-890.

12 S. E. Kentish, C. A. Scholes and G. W. Stevens, Recent Pat. Chem. Eng., 2010, 1, 52-66.

13 P. S. Goh, A. F. Ismail, S. M. Sanip, B. C. Ng and M. Aziz, Sep. Purif. Technol., 2011, 81, 243-264.

14 H. B. Tanh Jeazet, C. Staudt and C. Janiak, Dalton Trans., 2012, 41, 14003-14027.

15 D. Vu, J. Membr. Sci., 2003, 211, 311-334.

16 W. Rafizah and A. Ismail, J. Membr. Sci., 2008, 307, 53-61.

17 S. Kim, T. W. Pechar and E. Marand, Desalination, 2006, 192, 330-339.

18 S. Kim, L. Chen, J. K. Johnson and E. Marand, J. Membr. Sci., 2007, 294, 147-158.

19 S. Kim, J. R. Jinschek, H. Chen, D. S. Sholl and E. Marand, Nano Lett., 2007, 7, 2806-2811.

20 S. Kim and E. Marand, Microporous Mesoporous Mater., 2008, 114, 129-136.

21 B. D. Reid, F. A. Ruiz-Trevino, I. H. Musselman, K. J. Balkus and J. P. Ferraris, Chem. Mater., 2001, 13, 2366-2373.

22 T. Pechar, S. Kim, B. Vaughan, E. Marand, M. Tsapatsis, H. Jeong and C. Cornelius, J. Membr. Sci., 2006, 277, 195-202.

23 D. Sen, H. Kalipcilar and L. Yilmaz, J. Membr. Sci., 2007, 303, 194-203.

24 S. Tantekin-Ersolmaz, J. Membr. Sci., 2000, 175, 285-288.

25 S. Husain and W. J. Koros, J. Membr. Sci., 2007, 288, 195-207.

26 C. E. Powell and G. G. Qiao, J. Membr. Sci., 2006, 279, 1-49.

27 T. C. Merkel, Science, 2002, 296, 519-522.

28 T. C. Merkel, B. D. Freeman, R. J. Spontak, Z. He, I. Pinnau, P. Meakin and A. J. Hill, Chem. Mater., 2003, 15, 109-123.

29 L. Huang, Z. Wang, J. Sun, L. Miao, Q. Li, Y. Yan and D. Zhao, J. Am. Chem. Soc., 2000, 122, 3530-3531.

30 M. Smaïhi, E. Gavilan, J.-O. Durand and V. P. Valtchev, J. Mater. Chem., 2004, 14, 1347-1351.

31 Y. Dai, J. R. Johnson, O. Karvan, D. S. Sholl and W. J. Koros, J. Membr. Sci., 2012, 401-402, 76-82.

32 L. M. Vane, V. V. Namboodiri and T. C. Bowen, J. Membr. Sci., 2008, 308, 230-241.
33 R. Mahajan and W. J. Koros, Polym. Eng. Sci., 2002, 42, 14201431.

34 H. Yong, J. Membr. Sci., 2001, 188, 151-163.

35 E. Karatay, H. Kalıpçılar and L. Yılmaz, J. Membr. Sci., 2010, 364, 75-81.

36 I. F. J. Vankelecom, S. Van den broeck, E. Merckx, H. Geerts, P. Grobet and J. B. Uytterhoeven, J. Phys. Chem., 1996, 100, 3753-3758.

37 R. Mahajan and W. J. Koros, Ind. Eng. Chem. Res., 2000, 39, 2692-2696.

38 S. A. Hashemifard, A. F. Ismail and T. Matsuura, J. Colloid Interface Sci., 2011, 359, 359-370.

39 J. Lee, O. K. Farha, J. Roberts, K. A. Scheidt, S. T. Nguyen and J. T. Hupp, Chem. Soc. Rev., 2009, 38, 1450-1459.

40 L. E. Kreno, K. Leong, O. K. Farha, M. Allendorf, R. P. Van Duyne and J. T. Hupp, Chem. Rev., 2012, 112, 1105-1125.

41 O. Kahn, Acc. Chem. Res., 2000, 33, 647-657.

42 C. G. Silva, A. Corma and H. García, J. Mater. Chem., 2010, 20, 3141-3156.

43 P. Horcajada, C. Serre, G. Maurin, N. A. Ramsahye, F. Balas, M. Vallet-Regí, M. Sebban, F. Taulelle and G. Férey, J. Am. Chem. Soc., 2008, 130, 6774-6780.

44 J.-R. Li, R. J. Kuppler and H.-C. Zhou, Chem. Soc. Rev., 2009, 38, 1477-1504.

45 J. Cravillon, S. Münzer, S. Lohmeier, A. Feldhoff, K. Huber and M. Wiebcke, Chem. Mater., 2009, 1410-1412.

46 J. A. Thompson, K. W. Chapman, W. J. Koros, C. W. Jones and S. Nair, Microporous Mesoporous Mater., 2012, 158, 292-299.

47 Q. Song, S. K. Nataraj, M. V. Roussenova, J. C. Tan, D. J. Hughes, W. Li, P. Bourgoin, M. A. Alam, A. K. Cheetham, S. A. Al-Muhtaseb and E. Sivaniah, Energy Environ. Sci., 2012, 5, 8359-8369.

48 T. Li, Y. Pan, K.-V. Peinemann and Z. Lai, J. Membr. Sci., 2013, 425-426, 235-242.

49 T. Yang, Y. Xiao and T.-S. Chung, Energy Environ. Sci., 2011, 4, 4171.

50 B. Seoane, V. Sebastián, C. Téllez and J. Coronas, CrystEngComm, 2013, 68, 1-5.

51 M. J. C. Ordoñez, K. J. Balkus Jr, J. P. Ferraris and I. H. Musselman, J. Membr. Sci., 2010, 361, 28-37.

52 S. Sridhar, R. S. Veerapur, M. B. Patil, K. B. Gudasi and T. M. Aminabhavi, J. Appl. Polym. Sci., 2007, 106, 1585-1594.

53 Y. Li, F. Liang, H. Bux, W. Yang and J. Caro, J. Membr. Sci., 2010, 354, 48-54.

54 B. Zornoza, C. Tellez, J. Coronas, J. Gascon and F. Kapteijn, Microporous Mesoporous Mater., 2013, 166, 67-78.

55 B. Zornoza, A. Martinez-Joaristi, P. Serra-Crespo, C. Tellez, J. Coronas, J. Gascon and F. Kapteijn, Chem. Commun., 2011, 47, 9522-9524.

56 E. Stavitski, E. A. Pidko, S. Couck, T. Remy, E. J. M. Hensen, B. M. Weckhuysen, J. Denayer, J. Gascon and F. Kapteijn, Langmuir, 2011, 27, 3970-3976.

57 S. Couck, J. F. M. Denayer, G. V. Baron, T. Rémy, J. Gascon and F. Kapteijn, J. Am. Chem. Soc., 2009, 131, 6326-6327.

58 A. L. Khan, S. Basu, A. Cano-Odena and I. F. J. Vankelecom, J. Membr. Sci., 2010, 354, 32-39. 
59 C. Zhang, Y. Dai, J. R. Johnson, O. Karvan and W. J. Koros, J. Membr. Sci., 2011, 389, 34-42.

60 K. Kida, M. Okita, K. Fujita, S. Tanaka and Y. Miyake, CrystEngComm, 2013, 15, 1794.

61 T. Rodenas, I. Luz, G. Prieto, B. Seoane, H. Miro, A. Corma, F. Kapteijn, F. X. Llabrés i Xamena and J. Gascon, Nat. Mater., 2015, 14, 48-55.

62 J. Sánchez-Laínez, B. Zornoza, S. Friebe, J. Caro, S. Cao, A. Sabetghadam, B. Seoane, J. Gascon, F. Kapteijn, C. Le Guillouzer, G. Clet, M. Daturi, C. Téllez and J. Coronas, J. Membr. Sci., 2016, 515, 45-53.

63 B. Zornoza, C. Casado and A. Navajas, in Renewable Hydrogen Technologies, Elsevier, 2013, pp. 245-268.

64 D. Fairen-Jimenez, R. Galvelis, A. Torrisi, A. D. Gellan, M. T. Wharmby, P. A. Wright, C. Mellot-Draznieks and T. Düren, Dalton Trans., 2012, 41, 10752.

65 L. Hertäg, H. Bux, J. Caro, C. Chmelik, T. Remsungnen, M. Knauth and S. Fritzsche, J. Membr. Sci., 2011, 377, 36-41.

66 E. Haldoupis, T. Watanabe, S. Nair and D. S. Sholl, ChemPhysChem, 2012, 13, 3449-3452.

67 D. Fairen-Jimenez, S. A. Moggach, M. T. Wharmby, P. A. Wright, S. Parsons and T. Düren, J. Am. Chem. Soc., 2011, 133, 8900-8902.
68 S. Aguado, G. Bergeret, M. P. Titus, V. Moizan, C. NietoDraghi, N. Bats and D. Farrusseng, New J. Chem., 2011, 35, 546-550.

69 M. R. Ryder, B. Civalleri, T. D. Bennett, S. Henke, S. Rudić, G. Cinque, F. Fernandez-Alonso and J.-C. Tan, Phys. Rev. Lett., 2014, 113, 215502.

70 R. Pal, J. Colloid Interface Sci., 2008, 317, 191-198.

71 R. F. Boyer and R. L. Miller, Macromolecules, 1977, 10, 11671169.

72 T. Rodenas, M. van Dalen, E. García-Pérez, P. Serra-Crespo, B. Zornoza, F. Kapteijn and J. Gascon, Adv. Funct. Mater., 2014, 24, 249-256.

73 M. C. McCarthy, V. Varela-Guerrero, G. V. Barnett and H.-K. Jeong, Langmuir, 2010, 26, 14636-14641.

74 H. Bux, F. Liang, Y. Li, J. Cravillon, M. Wiebcke and J. Caro, J. Am. Chem. Soc., 2009, 131, 16000-16001.

75 O. Shekhah, R. Swaidan, Y. Belmabkhout, M. du Plessis, T. Jacobs, L. J. Barbour, I. Pinnau and M. Eddaoudi, Chem. Commun., 2014, 50, 2089-2092.

76 Y.-S. Li, F.-Y. Liang, H. Bux, A. Feldhoff, W.-S. Yang and J. Caro, Angew. Chem., Int. Ed., 2010, 49, 548-551.

77 X. Y. Chen, V.-T. Hoang, D. Rodrigue and S. Kaliaguine, RSC Adv. , 2013, 3, 24266.

78 E. Ahmadi Feijani, A. Tavasoli and H. Mahdavi, Ind. Eng. Chem. Res., 2015, 54, 12124-12134. 